EPJ Web of Conferences 21, 01003 (2012)

DOI: $10.1051 /$ epjconf/20122101003

(C) Owned by the authors, published by EDP Sciences, 2012

\title{
Level Densities in the actinide region and indirect $n, y$ cross section measurements using the surrogate method
}

\author{
J.N. Wilson ${ }^{1, \mathrm{a}}$, F. Gunsing ${ }^{2}$, L. Bernstein ${ }^{4}$,A. Bürger ${ }^{3}$, A. Görgen ${ }^{3}$, M. Guttormssen ${ }^{3}$, A-C. Larsen ${ }^{3}$,

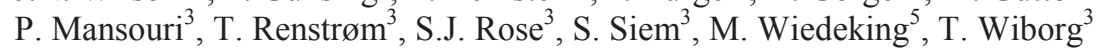 \\ ${ }^{1}$ Institut de Physique Nucléaire d'Orsay, Bât 100, 15 rue G. Clémenceau, 91406 Orsay, France \\ ${ }^{2}$ DSM/IRFU, CEA Saclay, Bât 141 , F-91191 Gif-sur-yvette Cedex, France \\ ${ }^{3}$ University of Oslo, Department of Physics, P.O. Box 1048, Blindern 0316 Oslo, Norway \\ ${ }^{4}$ Lawrence Livermoore National Laboratory, 7000 East Avenue, Livermore, CA 94550-9234, USA \\ ${ }^{5}$ Themba LABS,P.O. Box 722, 7129 Somerset West, South Africa
}

\begin{abstract}
.
Results from a program of measurements of level densities and gamma ray strength functions in the actinide region are presented. Experiments at the Oslo cyclotron involving the Cactus/Siri detectors and ${ }^{232} \mathrm{Th}(\mathrm{d}, \mathrm{x})$ and ${ }^{232} \mathrm{Th}\left({ }^{3} \mathrm{He}, \mathrm{x}\right)$ reactions were carried out to help answer the question of which level density model is the most appropriate for actinide nuclei, since it will have an impact on cross section calculations important for reactor physics simulations. A new technique for extracting level densities and gamma ray strength functions from particle-gamma coincidence data is proposed and results from the development of this technique are presented. In addition, simultaneous measurements of compound nuclear gamma decay probabilities have been performed for the key thorium cycle nuclei ${ }^{233} \mathrm{Th},{ }^{231} \mathrm{Th}$ and ${ }^{232} \mathrm{~Pa}$ up to around $1 \mathrm{MeV}$ above the neutron binding energy and have enabled extraction of indirect neutron induced capture cross sections for the ${ }^{232} \mathrm{Th},{ }^{231} \mathrm{~Pa}$ and

${ }^{230} \mathrm{Th}$ nuclei using the surrogate reaction method. Since the neutron capture cross section for ${ }^{232} \mathrm{Th}$ is already well known from direct measurements a comparison provides a stringent test of the applicability of the surrogate technique in the actinide region.
\end{abstract}

\section{Introduction}

Knowledge of neutron induced cross sections is important for both the physics of nuclear reactors and for understanding astrophysical processes of elemental synthesis. Unfortunately for many nuclei cross sections are impossible or extremely difficult to measure due to the necessity of using highly radioactive targets. For unknown cross sections theoretical estimates can vary by up to an order of magnitude since calculations require information on level densities and gamma ray strength functions which can change rapidly with nucleon number due to nuclear structure effects. In the first section of this article we propose a new method for extracting level density and gamma ray strength function information which may help us to come to a systematic understanding of how level densities evolve with nucleon number in the actinide region and which model(s) are the most appropriate. This in turn may help improve cross section calculations for nuclei where no experimental information exists.

In the second section we present indirect neutron capture cross section measurements for thorium cycle nuclei using the surrogate method, a method developed to provide cross section information for nuclei where direct measurements are difficult or impossible.

The data presented in both sections of the article were obtained from experiments detecting charged particle gamma ray coincidences with the Silicon Ring[1] and Cactus gamma ray multidetector at the Oslo cyclotron using transfer reactions of ${ }^{232} \mathrm{Th}(\mathrm{d}, \mathrm{x})$ at $12 \mathrm{MeV}$ and ${ }^{232} \mathrm{Th}\left({ }^{3} \mathrm{He}, \mathrm{x}\right)$ at 24 $\mathrm{MeV}$.

a e-mail : wilson@ipno.in2p3.fr

This is an Open Access article distributed under the terms of the Creative Commons Attribution-Noncommercial License 3.0, which permits unrestricted use, distribution, and reproduction in any noncommercial medium, provided the original work is properly cited. 


\section{Extractions of Level Densities and Strength Functions via Monte Carlo Data Modelling}

Nuclear Level Densities (NLD) and gamma ray strength functions (GSF) are important nuclear properties that are difficult to access experimentally. Some partial information can be obtained from spectroscopy of low-lying states and neutron resonance spacing at the binding energy. At intermediate energies further information can be extracted using a method which has been developed over many years, called the Oslo method[2]. The method relies on collecting and processing raw data of particle-gamma coincidences obtained from transfer reactions. The energies of ingoing and outgoing charged particles are used to determine the resulting excitation energy of the target nucleus and the gamma-rays associated with its decay are then measured in a high efficiency gamma ray spectrometer.

The Oslo method relies on processing of the raw particle gamma coincidence data in a series of steps involving: 1) histogramming a complete set of gamma ray spectra as a function of the compound nuclear excitation energy; 2) the unfolding of the gamma detector response by an iterative unfolding procedure to obtain the spectra actually emitted from the excited nucleus; 3 ) finding the set of primary gamma spectra as a function of excitation energy by another iterative procedure; 4) extracting the level density and gamma ray strength function via a further iterative method. The extraction of NLD and GSF at the final step relies on a factorisation where the probability of a transition from an initial state $i$ to a final state $f$ is proportional to the final density of states (Fermi's golden rule)

$$
P\left(E_{i}-E_{f}\right)=\rho\left(E_{f}\right) T\left(E_{\gamma}\right)
$$

where the transmission coefficient $T\left(E_{\gamma}\right)$ is related to the gamma ray strength function, which for dipole radiation is :

$$
T(E \gamma)=1 /\left(2 \pi E_{\gamma}^{3}\right)
$$

In the factorization, the decay probability is also considered proportional to the gamma ray transition coefficient which is assumed to be independent of compound nuclear excitation energy and vary only as a function of gamma ray energy (the Brink hypothesis). The Oslo method has been used successfully to provide information on nuclear level densities and gamma ray strength functions in many different nuclei[3-6].

However potential drawbacks of the method are that it relies on processing the raw data through a series of three consecutive iterative procedures, each one involving achieving a solution through convergence. It is difficult to prove that at each step that the converged solution is the best possible or "correct" solution and even small errors occurring at any point in the data processing chain could magnify. Certain limitations and potential introduction of systematic errors for each individual step of the method are explored in depth in [2]. However, it is not completely understood how statistical errors from the raw data and systematic errors from the detector response functions, and the effect of finite detector resolution, etc. propagate through the complex series of data processing steps and affect the final results.

We therefore propose a new method for extracting NLD and GSF which involves working backwards and is intended as a complimentary procedure to the Oslo method. The method has the potential to be more robust since it requires just a single minimisation procedure to fit the experimental data in two dimensions. Instead of starting with the raw data and attempting to extract level densities and strength functions, we start with trial level densities and strength functions and calculate what the raw data would look like in a "simulated" experiment. In this way we can avoid any potential problems associated with convergence of iterative procedures and associated systematic and statistical errors.

The new method works as follows: Trial NLD and GSF functions are used as an initial input and a nuclear level spectrum is then calculated. A Monte-Carlo procedure then generates a large 


\section{$\mathrm{CNR} * 11$}

number of gamma cascades from randomly chosen initial states to the ground state. Thus the resulting set of emitted gamma ray spectra as a function of compound nuclear excitation energy is generated. Since the response function of the Cactus multi-detector is well known, the set of simulated detected spectra are then produced (see fig. 1.). The resulting matrix (fig. 2) is then normalized and can be compared with the existing experimental data by computing the sum of the square of the differences between each bin in the $2 \mathrm{D}$ histograms. The result is a single value, $\chi^{2}$, which represents the "goodness of fit" or degree of similarity between the simulated and experimental data. An iterative $\chi^{2}$ minimisation procedure using standard computational techniques can then be performed on the free parameters which define the trial NLD and GSF functions.

The potential major disadvantage is the speed of the computation. Currently a $2 \mathrm{D}$ histogram ( $10 \mathrm{keV} / \mathrm{channel}$ bin size) of simulated data based on the simulated emission of $10^{7}$ cascades can be produced using around 10 seconds of computer time on a $2.8 \mathrm{GHz}$ dual quad core $(8 \mathrm{CPU})$ machine. The minimisation procedure may require up to $10^{5}$ function evaluations depending on the number of degrees of freedom in the trial NLD and GSF functions, or approximately 10 days of computer time.

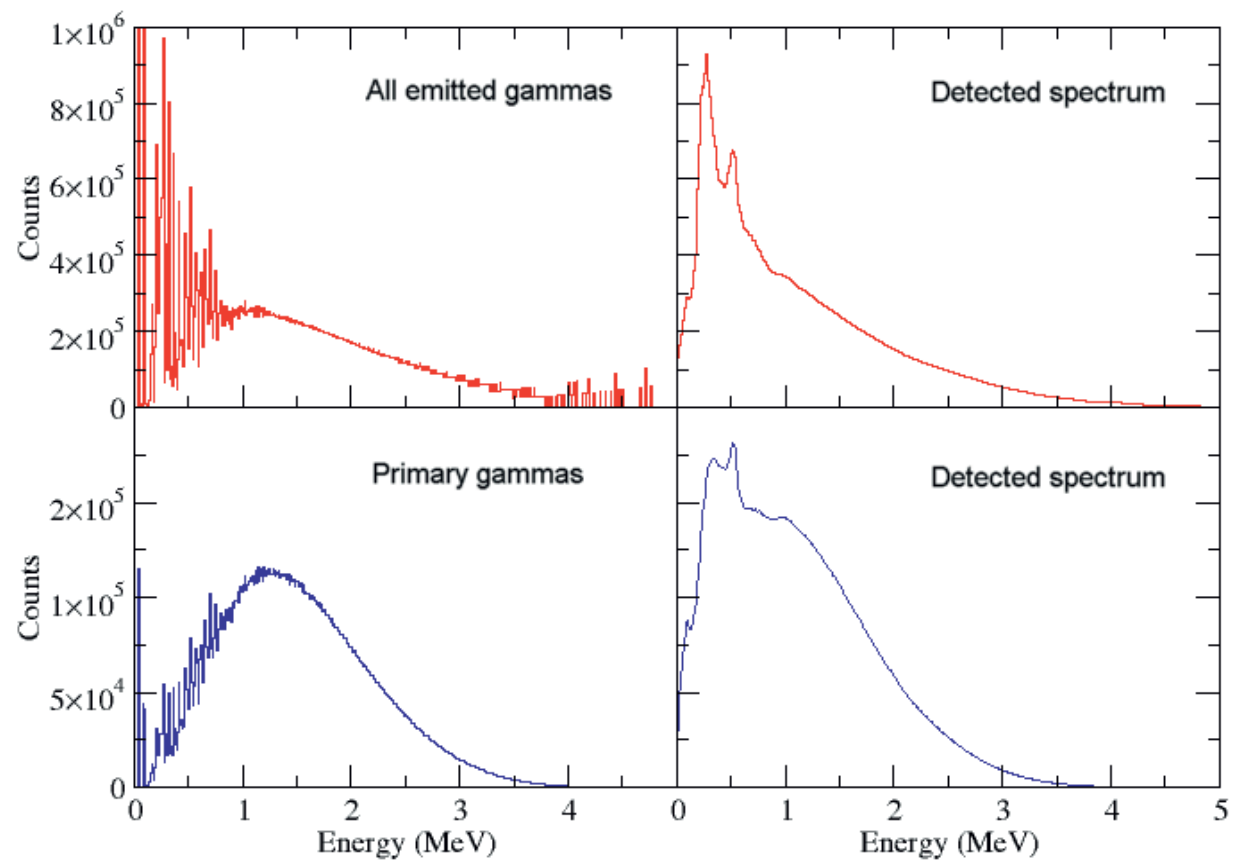

Fig. 1. Monte-Carlo simulated emitted and detected gamma ray spectra for cascades starting at 4.78 $\mathrm{MeV}$. In this case a constant temperature level density model with level density parameter of $\mathrm{a}=21$ $\mathrm{MeV}^{-1}$ and a gamma ray strength function which did not vary with energy. 


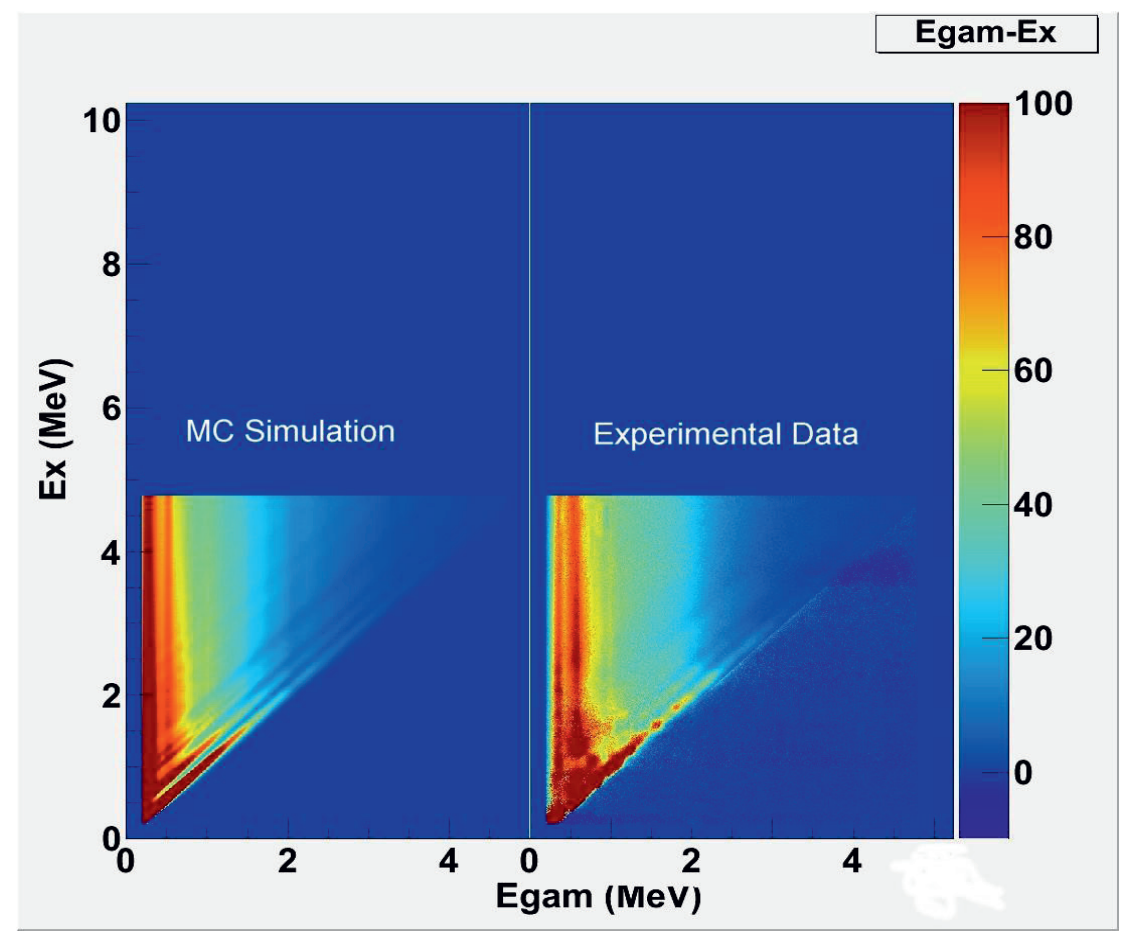

Fig. 2. Comparison of the Monte-Carlo generated simulated data and the experimental data.

A comparison between NLD and GSF extracted using the traditional Oslo method and the new Monte-Carlo based method will be performed in the near future. It remains to be seen how many free parameters need to be used in the new method to reproduce the raw experimental data within the statistical errors. Obviously the more free parameters introduced, the better the fit will be. However, introducing too many free parameters will increase the number of spatial dimensions in the minimisation procedure. This in turn will require a greater number of function evaluations and could hinder convergence to the best solution. Research on this method is ongoing.

\section{Indirect Measurements using the Surrogate Method}

The surrogate method was proposed in the 1970's to address the difficulty of performing direct cross section measurements on short-lived, highly radioactive targets[7]. A transfer reaction involving charged particles in the entry and exit channels is substituted for a direct neutron induced reaction. If the energy of the ingoing and outgoing particles is known, then the excitation energy of the final nucleus can be deduced and a probability of subsequent decay can be measured. The method relies on the Weisskopf-Ewing approximation that a particular cross section can be broken into two parts, one relating to the formation cross section of the compound nucleus $\sigma_{C N}(E)$ and the other a probability of decay into a particular reaction channel $P(E)$, e.g. fission, capture, etc. (see eqn. 3).

$$
\sigma_{(n, \gamma)}(E)=\sigma_{C N}(E) P(E)
$$




\section{$\mathrm{CNR} * 11$}

The approximation is valid if two conditions any differences in spin distributions of the direct and indirect reactions have no effect on the decay probability. In practice this may not be true and needs to be tested. The decay probability is difficult to calculate because it depends strongly on nuclear structure effects but can be measured using a surrogate reaction. The compound nucleus formation cross section can be easily calculated with the optical model and thus the cross section information can be extracted.

While it is generally accepted that the surrogate method appears to reliably reproduce known fission cross sections[8], recent results by Scielzo et al.[9] in gadolinium nuclei indicate that for capture cross sections it leads to a large overestimation of up to $400 \%$. Their explanation for these results is that the Weisskopf-Ewing approximation (that spin differences between direct and indirect reactions don't matter) is inapplicable for capture cross sections and that the spin distribution of the compound nucleus strongly affects the decay probability.

Recently we have performed surrogate capture cross section measurements for three nuclei in the actinide region ${ }^{232} \mathrm{Th}(\mathrm{n}, \gamma),{ }^{231} \mathrm{~Pa}(\mathrm{n}, \gamma)$ and ${ }^{230} \mathrm{Th}(\mathrm{n}, \gamma)[10]$. The experiments were performed at the Oslo Cyclotron using the Cactus gamma-ray detector coupled with the Silicon Ring charged particle array and decay probabilities above the neutron binding energy were measured using the weighting function technique[11]. Since the ${ }^{232} \mathrm{Th}(\mathrm{n}, \gamma)$ cross section is well known from recent direct neutroninduced measurements at the nTOF facility[12], the validity of the surrogate method for capture cross sections in the actinide region has been tested for the first time.

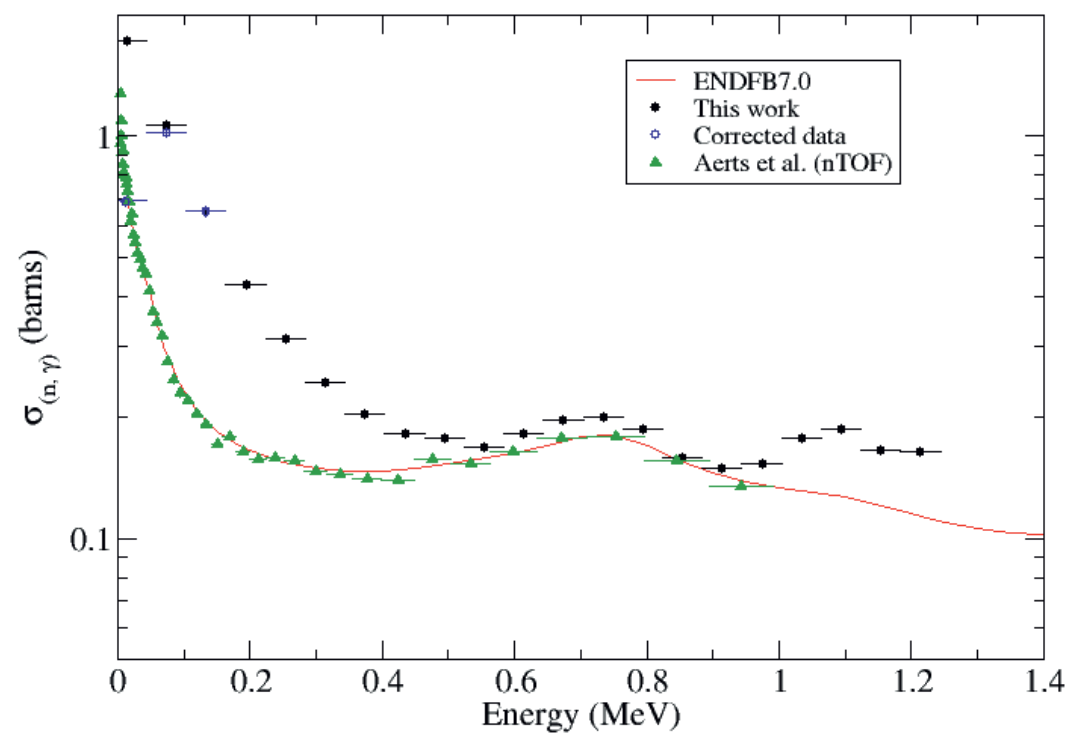

Fig. 3. Comparison of the indirect (surrogate method) and direct capture cross section measurements for the $232 \mathrm{Th}$ (n,gamma) cross section. Between $500 \mathrm{keV}$ and $1 \mathrm{MeV}$ there is reasonable agreement between the data. At low energies the surrogate method leads to a large overestimation of the cross section.

The comparison of results can be seen in fig 3., which shows that below $500 \mathrm{keV}$ we observe similar large overestimated of the cross section ( $400 \%)$ similar to those seen in the Lanthanides. However, between $500 \mathrm{keV}$ and $1 \mathrm{MeV}$ the direct and indirect experiments are in acceptable agreement of around $15 \%$. Above the fission threshold at $1 \mathrm{MeV}$ gamma rays from fission begin to contaminate our spectra as the leading to an understandable increase in the indirectly measured cross section. Fission detectors were not present in the experimental setup but in future experiments we plan to include fission detectors so that the contribution from fission gammas can be subtracted. 
EPJ Web of Conferences

\subsection{Conclusion}

An explanation for the cross section overestimation observed in the lanthanides has been proposed in [13]. The principal cause of the capture cross section overestimation using the surrogate method arises from neutron emission being hindered at higher spins and low excitations above the binding energy due to a lack of suitable final states for the decay since the neutron is a spin $1 / 2$ particles and cannot carry away much angular momentum. In Lanthanide nuclei the levels in the A-1 system are spaced too far apart and are at too low spin to act as final states for the neutron decay if the compound nucleus excitation energy is too close to the binding energy and it has too much spin. Thus the gamma decay dominates since the neutron emission is blocked and the capture cross section is overestimated by a large factor.

The situation for our ${ }^{232} \mathrm{Th}$ results is mixed, with overestimation at low energies and agreement at high energies. This implies that there is a domain of validity for surrogate reactions where the Weisskopf-Ewing approximation applies. The exact extent of this domain of validity remains to be uncovered. It is, however, possible to suggest some general trends. The domain of validity will likely depend on the following: (i) the size of the spacing of the lowest energy levels and the ground state spin in the A-1 system with lowest level spacing found in the highest mass nuclei, (ii) the excitation energy above the neutron binding energy in the compound system, (iii) the particular surrogate reaction used with projectiles of the highest masses producing the greatest spin differences. In general surrogate measurements may only provide an upper limit on cross sections rather than precise values. The explanation of neutron emission blocking proposed in [13] poses a further problem, since if it is correct then it should also apply to fission since neutron emission also competes with fission. It may well be worth testing the surrogate method for fission cross sections at low energies to see if the same overestimation occurs. Further research on the extent of the domain of validity of the surrogate method is therefore clearly necessary.

\section{References}

1. M. Guttormsen, A Bürger, T.E. Hansen and N. Liater, Nucl. Instr. Meth. A Submitted (2011)

2. A.C. Larsen et al., Phys. Rev. C 83034315 (2011)

3. A. Schiller et al., Phys. Lett. B 633, 225 (2006)

4. E. Melby et al., Phys. Rev. Lett. 833150 (1999)

5. U. Agvaanluvansan et al., Phys. Rev. Lett. 102162504 (2009)

6. A. Voinov et al., Phys. Rev. Lett. 93142504 (2004)

7. J. Cramer and H. Britt, Nucl. Sci. Eng. 41177 (1970)

8. G. Kessedjian et al., Phys. Lett. B 692297 (2010)

9. N. Scielzo et al., Phys. Rev. C 81034608 (2010)

10. J.N. Wilson et al., Nucl. Instr. Meth. A511 388 (2003)

11. J.N. Wilson et al., Phys. Rev. C. Submitted (2011)

12. G.Aerts et al., Phys. Rev. C 73054610 (2006)

13. G. Boutoux, PhD. Thesis, CENBG Bordeaux (2011) 